\title{
Physical activity assessed by activity monitor and doubly labeled water in children.
}

Citation for published version (APA):

Ekelund, U., Sjostrom, M., Yngve, A., Poortvliet, E., Nilsson, A., Froberg, K., Wederkopp, N., \&

Westerterp, K. R. (2001). Physical activity assessed by activity monitor and doubly labeled water in children. Medicine and Science in Sports and Exercise, 33, 275-281. https://doi.org/10.1097/00005768200102000-00017

Document status and date:

Published: 01/01/2001

DOI:

10.1097/00005768-200102000-00017

Document Version:

Publisher's PDF, also known as Version of record

Document license:

Taverne

Please check the document version of this publication:

- A submitted manuscript is the version of the article upon submission and before peer-review. There can be important differences between the submitted version and the official published version of record.

People interested in the research are advised to contact the author for the final version of the publication, or visit the DOI to the publisher's website.

- The final author version and the galley proof are versions of the publication after peer review.

- The final published version features the final layout of the paper including the volume, issue and page numbers.

Link to publication

\footnotetext{
General rights rights.

- You may freely distribute the URL identifying the publication in the public portal. please follow below link for the End User Agreement:

www.umlib.nl/taverne-license

Take down policy

If you believe that this document breaches copyright please contact us at:

repository@maastrichtuniversity.nl

providing details and we will investigate your claim.
}

Copyright and moral rights for the publications made accessible in the public portal are retained by the authors and/or other copyright owners and it is a condition of accessing publications that users recognise and abide by the legal requirements associated with these

- Users may download and print one copy of any publication from the public portal for the purpose of private study or research.

- You may not further distribute the material or use it for any profit-making activity or commercial gain

If the publication is distributed under the terms of Article $25 \mathrm{fa}$ of the Dutch Copyright Act, indicated by the "Taverne" license above, 


\title{
Physical activity assessed by activity monitor and doubly labeled water in children
}

\author{
ULF EKELUND, MICHAEL SJÖSTRÖM, AGNETA YNGVE, ERIC POORTVLIET, ANDREAS NILSSON, \\ KARSTEN FROBERG, NIELS WEDDERKOPP, and KLAAS WESTERTERP
}

Unit for Preventive Nutrition, Department of Medical Nutrition, Karolinska Institutet, Stockholm, SWEDEN; Department of Physical Education and Health, Örebro University, Örebro, SWEDEN; Institute of Sport Science and Clinical Biomechanics, University of Southern Denmark, Odense, DENMARK; and Department of Human Biology, Maastricht University, Maastricht, THE NETHERLANDS

\begin{abstract}
EKElund, U., M. SJÖSTRÖM, A. YNGVE, E. POORTVLIET, A. NILSSON, K. FROBERG, N. WEDDERKOPP, and K. WESTERTERP. Physical activity assessed by activity monitor and doubly labeled water in children. Med. Sci. Sports Exerc., Vol. 33, No. 2, pp. 275-281, 2001. Purpose: To validate the Computer Science and Application's (CSA) activity monitor for assessment of the total amount of physical activity during two school-weeks in 9-yr-old children and to develop equations to predict total energy expenditure (TEE) and activity energy expenditure (AEE) from activity counts and anthropometric variables. Methods: A total of 26 children ( 15 boys and 11 girls, mean age $9.1 \pm 0.3 \mathrm{yr}$ ) were monitored for 14 consecutive days. TEE was simultaneously measured by the doubly labeled water method. Averaged activity counts (counts $\cdot \mathrm{min}^{-1}$ ) were compared with data on: 1 ) TEE, 2) AEE $=$ TEE minus basal metabolic rate (BMR; estimated from predictive equations), and 3) daily physical activity level (PAL = TEE/BMR). Results: Physical activity determined by activity counts was significantly related to the data on energy expenditures: TEE $(r=0.39$; $P<0.05)$, AEE $(\mathrm{r}=0.54 ; P<0.01)$, and PAL $(\mathrm{r}=0.58 ; P<0.01)$. Multiple stepwise regression analysis showed that TEE was significantly influenced by gender, body composition (body weight or fat free mass), and activity counts $\left(\mathrm{R}^{2}=0.54-0.60\right)$. AEE was significantly influenced by activity counts and gender $\left(R^{2}=0.45\right)$. There were no significant differences between activity counts and PAL in discriminating among activity levels with "low" (PAL < 1.56), "moderate" (1.57 $\leq$ PAL $\geq 1.81)$, and "high" (PAL > 1.81) intensity. Conclusion: Activity counts from the CSA activity monitor seems to be a useful measure of the total amount of physical activity in 9-yr-old children. Activity counts contributed significantly to the explained variation in TEE and was the best predictor of AEE. Key Words: ACCELEROMETER, DAILY ACTIVITY, ENERGY EXPENDITURE
\end{abstract}

$\mathrm{A}$ ccurate assessment of the total amount of children's physical activity, or the energy expended, is critically important when examining the relationship between physical activity and health (e.g., cardiovascular disease risk factors, fatness, and aerobic fitness). Until now, self-reported methods have been used, such as recall interview and questionnaires $(3,18)$. The self-report methods rely on the subject's ability to recall and report physical activity and should be used with caution, especially in subjects younger than $15 \mathrm{yr}$ of age (20). Furthermore, selfreport methods have not been validated, with few exceptions (5), against the doubly labeled water (DLW) method, which is considered to be the "golden standard" for the determination of total energy expenditure (TEE) under free-living conditions.

The DLW method measures TEE over longer periods (1-3 wk) and therefore provides a good estimate of average daily TEE $(17,23,29)$. The accuracy for TEE measurements is $4-7 \%$ (22). Together with an estimate of basal metabolic rate (BMR), the energy expenditure for physical activity

0195-9131/01/3302-0275/\$3.00/0

MEDICINE \& SCIENCE IN SPORTS \& EXERCISE ${ }_{\circledast}$

Copyright (C) 2001 by the American College of Sports Medicine

Submitted for publication December 1999.

Accepted for publication May 2000. (activity energy expenditure; AEE $=$ TEE - BMR) can be calculated. Physical activity level (PAL) can be calculated by expressing TEE as a multiple of BMR (PAL = TEE/ BMR). However, the high cost of the stable isotopes and the sophisticated analysis technique limits the usefulness of the DLW method in epidemiological studies.

To reduce the errors associated with self-report methods in children, objective methods such as motion sensors have been developed. The Computer Science and Applications Inc. (CSA) uni-axial activity monitor (WAM 7164; Computer Science and Application's Inc., Shalimar, FL) is one of these. Its small size $(5.1 \times 3.8 \times 1.5 \mathrm{~cm}$ and $43 \mathrm{~g})$, robust design, and social acceptability seem to make it suitable for use in largescale epidemiological studies in children and adolescents (28). It has previously been validated against energy expenditure (EE) measured by indirect calorimetry under laboratory conditions $(7,9,27)$. It was found to be a valid tool for quantifying $\mathrm{EE}$ in 10- to 14-yr-old children during walking and running on a treadmill (27). A high correlation $(r=0.88)$ has been found between steady-state EE and activity counts in adults (9). Activity counts have been compared with scaled oxygen uptake $\left(\mathrm{V}_{2} \cdot \mathrm{kg}^{-0.75} \cdot \mathrm{min}^{-1}\right)$ in 9-yr-old children during standardized (treadmill walking and running) and free-playing (playing catch, hopscotch, sitting, and crayoning) activities (7). Correlation coefficients between CSA activity counts and $\dot{\mathrm{V}} \mathrm{O}_{2} \cdot \mathrm{kg}^{-0.75} \cdot \mathrm{min}^{-1}$ were $0.78,0.69$, and 0.85 for all activities, 
treadmill activities, and free-playing activities, respectively. Although the results from these controlled research settings seems promising, it is not clear whether the CSA activity monitor can be used for valid prediction of the total amount of physical activity and TEE under free-living conditions in children. The aim of the present study was to evaluate the validity of the CSA activity monitor, using the DLW method as criterion measure, in 9-yr-old children. A secondary aim was to develop regression equations to predict TEE and AEE using activity counts and anthropometric variables.

\section{METHODS}

\section{Subjects, Selection, and Study Design}

The subjects were selected through a two-stage sampling procedure. First, four schools, in the community of Odense, Denmark, were selected at random. Second, 60 randomly selected children from these four schools were invited to participate. Of these, 29 (17 boys and 12 girls) agreed to participate. Two of the children, or their parents, were not willing to participate in the stable isotope part (DLW measurements) of the study, and data from one boy had to be excluded due to malfunction of the activity monitor. Therefore, the data reported here include 26 subjects ( 15 boys and 11 girls). The study was approved by the ethic committee of Vejle and Funen. Written and verbal informed consent was obtained from all parents and children. The study was carried out during two school-weeks in February. A schematic presentation of the study protocol is shown in Figure 1.

\section{Measurements}

Anthropometric measurements. Height was measured to the nearest $0.5 \mathrm{~cm}$ with a transportable Harpenden stadiometer. Body weight was measured by a calibrated beam balance to the nearest $0.1 \mathrm{~kg}$. Body fat was calculated from triceps and subscapula skinfolds (25) using a Harpenden skinfold caliper on the left side of the body. All measures were made in duplicate, and the average of these was used in the analysis. The same assistant performed all

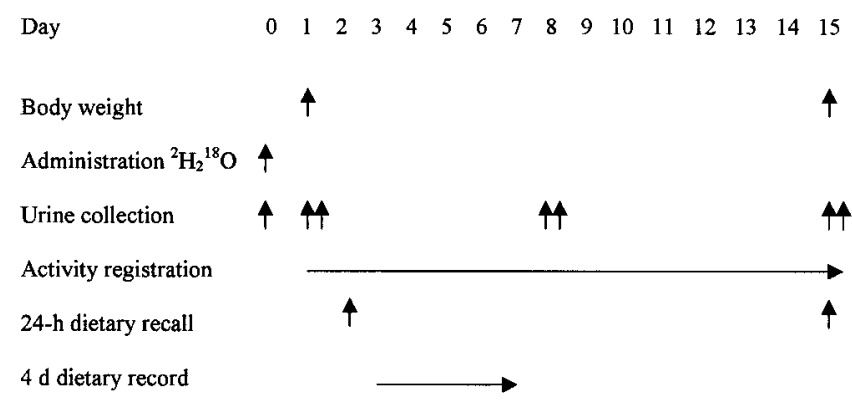

Figure 1-A schematic representation of the experimental protocol. The protocol consisted of a 14-d period during which the children were following their normal living. Physical activity was measured by the CSA activity monitor, and TEE was simultaneously measured by the DLW method from day 1 to day 15. Body weight was examined on day 1 and day 15 to investigate energy balance. Two different dietary intake methods (4-d weighted record and a $24-h$ recall) were performed during the 14-d period. of the skinfold measurements. Body fat mass was obtained by multiplying percentages of body fat by body weight. Fat-free mass was calculated by subtracting fat mass from body weight. Total body water was measured by deuterium dilution $\left({ }^{2} \mathrm{H}\right)$ as described by Westerterp et al. (30). The ${ }^{2} \mathrm{H}$ dilution space was divided by 1.04 to derive total body water. Fat mass and fat-free mass were calculated from total body water assuming a hydration factor for fat-free mass of $76.6 \%$ and $74.9 \%$ for boys and girls, respectively (8). The ratio of ${ }^{2} \mathrm{H}$ to oxygen dilution space was $1.048 \pm 0.013$.

Physical activity. The CSA activity monitor measures accelerations from 0.05 to $2.1 \mathrm{G}$. It is equipped with a 0.1 to 3.6 passband filter, which discriminates human movement from vibrations. The output from the monitor is sampled 10 times per second and summed over a selected time interval (epoch) (28). The memory capacity is $22 \mathrm{~d}$ by using a 60-s epoch. The monitor was secured directly to the skin as close as possible to the center of gravity (i.e., lower back) using an elastic belt. It was initialized as described by the manufacturer (6), and the 60-s epoch was used. The children and their parents were carefully instructed, verbally and in writing, on how to attach the activity monitor during each day of the whole measurement period (i.e., for 14 consecutive days) (Fig. 1). The children were asked to wear the monitor during the daytime. Exceptions were during water activities such as swimming and bathing. In addition, the subjects and their parents were asked to record the time when the monitor was attached and removed each day, which allowed calculation of registered activity time and sleeping time. The total amount of physical activity from the activity monitor was expressed as the average of total counts per minute of registered time.

TEE. TEE was measured over the 2 -wk period using the DLW method (Fig. 1). Dose, sampling protocol, sample analysis, and calculation procedure have been described elsewhere (30). A weighted dose of water with a measured enrichment of about 5 atoms $\%^{2} \mathrm{H}$ and 10 atoms $\%{ }^{18} \mathrm{O}$ was ingested. This dose increases the baseline levels (PPM) with 150 for ${ }^{2} \mathrm{H}$ and 300 for ${ }^{18} \mathrm{O}$. A baseline urine sample was collected before dosing as well as from the second and last voidings of the following day and from days 8 and 15 . Samples were analyzed in duplicate with an isotope-ratio mass spectrometer (Aqua Sira; VG Isogas, Middlewich, Cheshire, England). $\mathrm{CO}_{2}$ production was calculated from the elimination rates of the isotopes, as calculated from the slope of the elimination curve, correcting for changes in body water assumed to be proportional to changes in body mass from day 1 to day $15 . \mathrm{CO}_{2}$ production was converted to TEE using an energy equivalent based on the individual food quotient (FQ) calculated from the macronutrient composition of the diet as described by Black et al.(1), assuming RQ to be equal to FQ. A 4-d weighed dietary record was used for calculation of energy intake (EI) (Fig. 1).

BMR. Due to practical reasons (data collection was school-based), BMR was predicted from gender, age, weight, and height (24).

AEE. Assuming that $10 \%$ of TEE was due to the diet induced thermogenesis (DIT) (15), the energy expenditure 
associated with physical activity was calculated as TEE $($ BMR + TEE $\times 0.1)$. Furthermore, the PAL was calculated by expressing TEE as a multiple of BMR.

\section{Statistics}

The characteristics of subjects were described by using mean and SD. Differences between gender groups for physical characteristics and energy expenditure measurements were tested by one-way ANOVA.

Univariate relationship. Pearson correlation coefficients were computed to determine the linear relationship between activity counts (counts. $\mathrm{min}^{-1}$ ) and TEE, AEE, and PAL as well as between activity counts (counts. $\mathrm{min}^{-1}$ ) and the physical characteristic variables (body weight, fat-free mass, fat mass, and height). Partial correlations were computed to determine the relationship between activity counts and TEE and AEE after adjusting for gender and body weight.

Multivariate relationship. Stepwise multiple regression analysis was used to determine which of the independent variables contributed to the variation in TEE and AEE. Independent variables included height, body weight, fat-free mass, activity counts (counts. $\mathrm{min}^{-1}$ ), and gender. In all analyses for gender, girls were coded $=0$ and boys $=1$. TEE and AEE prediction equations were developed in a random sample of 18 children and cross-validated in the remaining group of eight. To test the validity of these equations, the degree of agreement between predicted and DLW measured TEE and AEE were tested by the method described by Bland and Altman (2).

Trost et al. (27) developed a regression equation for the prediction of energy expenditure (EE) from activity counts as follows:

$\mathrm{EE}\left(\mathrm{kcal} \cdot \mathrm{min}^{-1}\right)=0.0008\left(\right.$ counts $\left.\cdot \mathrm{min}^{-1}\right)+0.08$ (body weight in $\left.\mathrm{kg}\right)-2.23$

This equation was used for calculation of EE during the daytime (i.e., registered time). During nonregistered time (i.e., sleep time), EE was assumed to equal estimated BMR. EE during awake time and during sleep time was summed and compared with TEE from the DLW method. Agreement between TEE predicted from the Trost et al. (27) equation and assessed by DLW was tested by a Bland and Altman plot (2).

The correspondence between the PAL from DLW and activity counts (counts. $\mathrm{min}^{-1}$ ) from CSA activity monitor in assigning subjects to different activity categories was analyzed with a Wilcoxon signed rank test. All statistical analyses were performed using SPSS (Statistical Package for the Social Sciences for Windows, 8.0, 1997; SPSS Inc., Chicago, IL). The level of statistical significance was set at $P<$ 0.05 .

\section{RESULTS}

\section{Physical Description, Energy Expenditures, and Activity Registration}

The physical characteristics and energy expenditure components are shown in Table 1. Significant differences be- tween gender were observed for fat mass $(P=0.014)$ and percentage of body fat $(P=0.005)$. There were no differences in TEE or any of the TEE components between boys and girls, except for TEE and AEE in relation to body weight $\left(\mathrm{kcal} \cdot \mathrm{kg}^{-1} \cdot \mathrm{d}^{-1}\right)$. Thirty-one percent and $28 \%$ of TEE referred to AEE for boys and girls, respectively. On average, the activity monitors were worn for $803 \pm 37 \mathrm{~min} \cdot \mathrm{d}^{-1}$.

\section{Correlations and Partial Correlations Between Activity Counts and Other Variables}

The relationships between data on activity counts and energy expenditure components are shown in Table 2. Activity counts (counts. $\mathrm{min}^{-1}$ ) were significantly associated with all energy expenditure components. After adjusting for gender and body weight, activity counts (counts. $\mathrm{min}^{-1}$ ) were significantly related to TEE (partial $\mathrm{r}=0.67 ; P<0.001$ ) and AEE (partial $\mathrm{r}=0.66 ; P<0.001$ ). The relationship between activity counts (counts. $\mathrm{min}^{-1}$ ) and AEE is shown in Figure 2.There were no significant associations between activity counts and any of the physical characteristics variables (weight, fat-free mass, fat mass, and height).

\section{Multivariate Analysis Explaining the Variation in TEE and AEE}

The determinants of TEE and AEE were examined by stepwise multiple regression analysis (Table 3). The first regression model included activity counts, gender, height, and body weight. TEE was significantly influenced by gender (adjusted $\mathrm{R}^{2}=0.12$ ) as well as activity counts and body weight, with a total model $\mathrm{R}^{2}$ of 0.60 . The second model included activity counts, gender, height, and FFM assessed by TBW. TEE was significantly influenced by FFM from TBW (adjusted $\mathrm{R}^{2}=0.24$ ) as well as gender and activity counts, with a total $\mathrm{R}^{2}$ of 0.54 . The third model included activity counts, gender, height, and FFM assessed by skin folds. TEE was significantly influenced by FFM from skin folds (adjusted $\mathrm{R}^{2}=0.13$ ) as well as gender and activity counts with a total $\mathrm{R}^{2}$ of 0.55 . The AEE regression model included activity counts, gender, height, and body weight. AEE was significantly influenced by activity counts

TABLE 1. Physical characteristics, energy expenditures, and activity counts of subjects by gender (mean $\pm S D$ ).

\begin{tabular}{lcc}
\hline & Boys $(\boldsymbol{N}=\mathbf{1 5})$ & Girls $(\boldsymbol{N}=\mathbf{1 1})$ \\
\hline Age $(\mathrm{y})$ & $9.1 \pm 0.3$ & $9.1 \pm 0.3$ \\
Weight $(\mathrm{kg})$ & $33.0 \pm 5.7$ & $37.0 \pm 5.0$ \\
Height $(\mathrm{m})$ & $1.40 \pm 0.06$ & $1.39 \pm 0.05$ \\
Fat-free mass $(\mathrm{kg})^{a}$ & $26.2 \pm 3.0$ & $26.9 \pm 2.7$ \\
Fat mass $(\mathrm{kg})^{a}$ & $6.7 \pm 3.0$ & $10.1 \pm 3.2^{*}$ \\
Body fat $(\%)$ & $19.6 \pm 5.6$ & $26.7 \pm 5.8 \dagger$ \\
TEE $\left(\mathrm{kcal} \cdot \mathrm{d}^{-1}\right)$ & $2122 \pm 275$ & $1973 \pm 198$ \\
TEE $\left(\mathrm{kcal} \cdot \mathrm{kg}^{-1} \cdot \mathrm{d}^{-1}\right)$ & $65.3 \pm 9.1$ & $54.2 \pm 8.2 \dagger$ \\
BMR $\left(\mathrm{kcal} \cdot \mathrm{d}^{-1}\right)$ & $1245 \pm 119$ & $1229 \pm 97$ \\
AEE $\left(\mathrm{kcal} \cdot \mathrm{d}^{-1}\right)$ & $664 \pm 192$ & $547 \pm 200$ \\
AEE $\left(\mathrm{kcal} \cdot \mathrm{kg}{ }^{-1} \cdot \mathrm{d}^{-1}\right)$ & $20.5 \pm 6.4$ & $15.3 \pm 6.1^{*}$ \\
PAL $(\mathrm{TEE} / \mathrm{BMR})$ & $1.71 \pm 0.2$ & $1.61 \pm 0.2$ \\
Activity counts $\left(\mathrm{cnts} \cdot \mathrm{min}^{-1}\right)$ & $626 \pm 98$ & $689 \pm 174$ \\
Registered time $\left(\mathrm{min}^{-1} \cdot \mathrm{d}^{-1}\right)$ & $805 \pm 38$ & $799 \pm 37$ \\
\hline
\end{tabular}

${ }^{a}$ Measured by total body water estimated from ${ }^{2} \mathrm{H}$ dilution.

${ }^{*} P<0.05$

$\dagger P<0.01$, boys vs girls. 
TABLE 2. Correlations and partial correlations between activity counts (counts·min $\left.{ }^{-1}\right)$ and TEE, AEE, and PAL in 9-yr-old children $(N=26)$.

\begin{tabular}{ccc}
\hline & $\begin{array}{c}\text { Correlation } \\
\text { With Activity } \\
\text { Counts }\end{array}$ & $\begin{array}{c}\text { Partial Correlation } \\
\text { With Activity } \\
\text { Counts (Adjusting } \\
\text { for Weight and } \\
\text { Gender) }\end{array}$ \\
\hline TEE $\left(\mathrm{kcal} \cdot \mathrm{d}^{-1}\right)$ & $0.39^{*}$ & $0.67 \ddagger$ \\
AEE $\left(\mathrm{kcal} \cdot \mathrm{d}^{-1}\right)$ & $0.54 \dagger$ & $0.66 \neq$ \\
$\mathrm{PAL}$ & $0.58 \dagger$ & \\
${ }^{*} P<0.05$. & & \\
$\dagger P<0.01$. & & \\
$\ddagger P<0.001$. & &
\end{tabular}

(adjusted $\mathrm{R}^{2}=0.16$ ) as well as gender with a total model of $\mathrm{R}^{2} 0.45$.

To test the validity of the developed TEE and AEE prediction equations, a cross-validation study was performed. Pairwise comparisons between predicted TEE and DLW measured TEE are shown in Figure 3A-C. The mean differences between measured and predicted TEE were -33 $\mathrm{kcal} \cdot \mathrm{d}^{-1}(P=0.80),-23 \mathrm{kcal} \cdot \mathrm{d}^{-1}(P=0.84)$, and -69 $\mathrm{kcal} \cdot \mathrm{d}^{-1}(P=0.58)$ for regressions 1,2 , and 3 , respectively. The $95 \%$ limits of agreement (mean $\pm 2 \mathrm{SD}$ ) were -731 $\mathrm{kcal} \cdot \mathrm{d}^{-1}$ to $665 \mathrm{kcal} \cdot \mathrm{d}^{-1}$ (regression 1), $-675 \mathrm{kcal} \cdot \mathrm{d}^{-1}$ to 629 $\mathrm{kcal} \cdot \mathrm{d}^{-1}$ (regression 2), and $-749 \mathrm{kcal} \cdot \mathrm{d}^{-1}$ to $611 \mathrm{kcal} \cdot \mathrm{d}^{-1}$ (regression 3). The same comparison was performed regarding AEE. The mean difference between measured and predicted AEE was $-45 \mathrm{kcal} \cdot \mathrm{d}^{-1}(P=0.58)$, and the $95 \%$ limits of agreement were $-485 \mathrm{kcal} \cdot \mathrm{d}^{-1}$ to $395 \mathrm{kcal} \cdot \mathrm{d}^{-1}$. No significant associations were observed between the mean and the difference for any of the plots, indicating that predicted TEE and AEE values showed similar individual variation throughout the range of data.

\section{Laboratory Predicted TEE in Relation to Measured TEE}

TEE predicted from the laboratory-derived relationship between energy expenditure and activity counts in combination with estimated BMR significantly underestimated

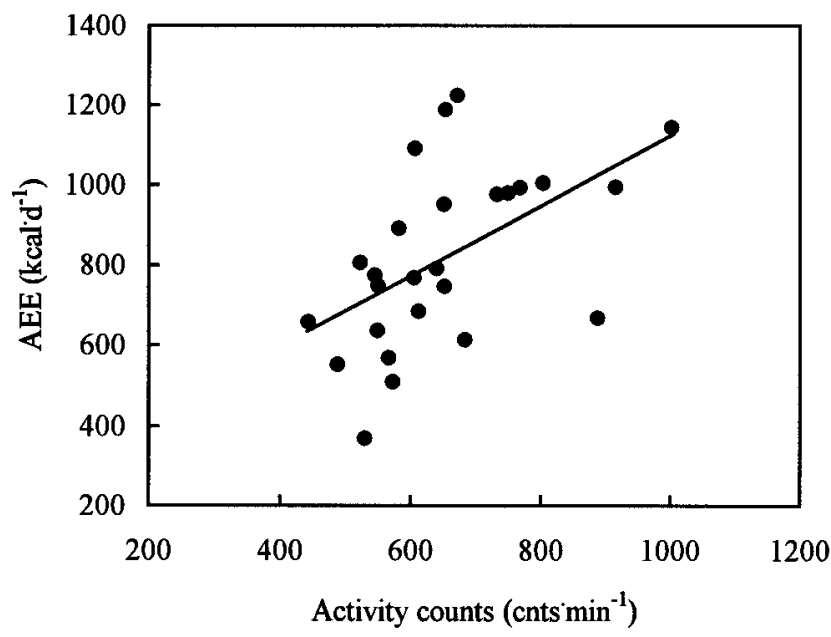

Figure 2-Relationship between activity energy expenditure $\left(\mathrm{kcal}^{-1} \mathrm{~d}^{-1}\right)$ and average activity counts (counts $\left.\cdot \mathrm{min}^{-1}\right)$. The solid line represents the regression line: $\mathrm{AEE}=0.874 \times$ activity counts $+250.3(N=26$; $\mathrm{r}=0.54 ; \mathrm{SEE}=192 ; P=0.005$ ).
TEE measured by the DLW method (mean difference -658 $\left.\pm 379 \mathrm{kcal} \cdot \mathrm{d}^{-1} ; P<0.001\right)$. The degree of agreement is shown in Figure 4. The 95\% limits of agreement were wide $\left(-1416\right.$ to $\left.100 \mathrm{kcal} \cdot \mathrm{d}^{-1}\right)$. There was a significant correlation ( $\mathrm{r}=0.48 ; P=0.01$ ) between the mean of the methods and the difference between the methods, indicating a higher degree of underestimation at low values for TEE.

\section{Correspondence Between Activity Counts and PAL}

To test the sensitivity of the CSA activity monitor to distinguish among different activity levels, the subjects were assigned to three equally sized groups corresponding to "low" $(N=8)$, "moderate" $(N=9)$, and "high" $(N=9)$ levels of physical activity based on their PAL from DLW and average activity counts (counts. $\mathrm{min}^{-1}$ ). The cut off values thus defined were as follows: low, PAL $<1.55$ and activity counts $<566$; moderate, $1.55 \geq \mathrm{PAL} \leq 1.80$ and $567 \geq$ activity counts $\leq 653$; and high, PAL $>1.80$ and activity counts $>653$. No significant difference (Wilcoxon signed rank test) was observed between the methods in discriminating between the three activity levels.

\section{DISCUSSION}

The purpose with the present study was to evaluate the validity of the CSA activity monitor for assessment of the total amount of physical activity in 9-yr-old children. Our data revealed that activity counts were significantly associated with all energy expenditure estimates from the DLW method. Furthermore, we found that total daily energy expenditure was significantly influenced by gender, body composition (weight or fat-free mass), and activity counts. Activity energy expenditure was significantly influenced by activity counts and gender.

An alternative uni-axial activity monitor, the Caltrac, significantly overestimated energy expenditure in physical activity (AEE) compared with AEE measured by DLW in 9-yr-old children (13). No significant relationship between AEE and "raw" activity counts from the Caltrac was found. The discrepancy between studies may be due to different activity monitors used because they may differ in their sensitivity in measuring vertical acceleration during free-living conditions. Both models of monitors have shown relatively high correlations $(\mathrm{r}=0.70$ 0.87 ) between activity counts and energy expenditure during specific activities in controlled laboratory settings $(7,19,27)$. Another possible explanation could be the number of days monitored ( $14 \mathrm{~d}$ compared with $3 \mathrm{~d}$ ). It has been concluded that $\geq 4 \mathrm{~d}$ of activity monitoring are needed to achieve satisfactory reliability in children (12). Bouten et al. (4) compared activity counts from a three-axial accelerometer (Tracmor) with energy expenditure estimates obtained by DLW in 30 adult subjects over $7 \mathrm{~d}$. An identical correlation coefficient $(\mathrm{r}=0.58 ; P<$ 0.001 ) as in the present study was found for the relationship between activity counts and PAL. After correction for activity counts arising from vibrations produced by transportation, the correlation was improved to 0.73 (4). More studies are needed 
TABLE 3. Stepwise regression analysis for estimating TEE $\left(\mathrm{kcal} \cdot \mathrm{d}^{-1}\right)$ and AEE $\left(\mathrm{kcal} \cdot \mathrm{d}^{-1}\right)$ from activity counts (counts·min $\left.{ }^{-1}\right)$ and anthropometric variables in 9-yr-old children $(N=18)$.

\begin{tabular}{|c|c|c|c|}
\hline & Prediction Equation & Adjusted $\mathrm{R}^{2}$ & SEE \\
\hline 1 & TEE $=(-$ Gender $\times 380.9)+($ Activity counts $\times 1.177)+($ Weight $(\mathrm{kg}) \times 21.1)+706$ & 0.60 & 150 \\
\hline 2 & TEE $=\left(\right.$ Fat-free mass $\left.{ }^{a}(\mathrm{~kg}) \times 41.6\right)-($ Gender $\times 277.6)+($ Activity counts $\times 0.808)+544$ & 0.54 & 162 \\
\hline 3 & TEE $=\left(\right.$ Fat-free mass $\left.{ }^{b}(\mathrm{~kg}) \times 39.4\right)-($ Gender $\times 334.4)+($ Activity counts $\times 0.876)+531.2$ & 0.55 & 159 \\
\hline 4 & $\mathrm{AEE}=($ Activity counts $\times 1.042)-($ Gender $\times 243.4)+238$ & 0.45 & 149 \\
\hline
\end{tabular}

Gender: Boys $=0 ;$ Girls $=1$

${ }^{a}$ Calculated from total body water measured by ${ }^{2} \mathrm{H}$ dilution.

${ }^{b}$ Calculated from the sum of two skinfolds (25).

to assess the total amount of physical activity using both uni-axial and tri-axial activity monitors simultaneously, preferably in comparison with energy expenditure estimates derived from DLW measurements.

The determinants of TEE and AEE were examined by multiple stepwise regression analysis. TEE was significantly influenced by gender, physical activity, and body composition (body weight or fat free mass). The best regression equation, explaining $60 \%$ of the variation in TEE, included gender, activity counts, and body weight. The two regression equations including fat-free mass, assessed by total body water and skin folds, explained together with gender and activity counts $54 \%$ and $55 \%$ of the variation in TEE, respectively. In a previous study performed in 4- to 10 -yr-old children, it was found that TEE was significantly influenced by body weight $\left(\mathrm{R}^{2}=\right.$ 0.65 ) as well as height, season, geographic location, and gender (total model $\mathrm{R}^{2}=0.68$ ) (11). Given the relatively homogenous sample regarding body weight in the present study compared with the study by Goran et al.(11), it is worth noting that as much as $60 \%$ of the variation in TEE was explained by gender, activity counts, and body weight. Furthermore, activity counts significantly con-
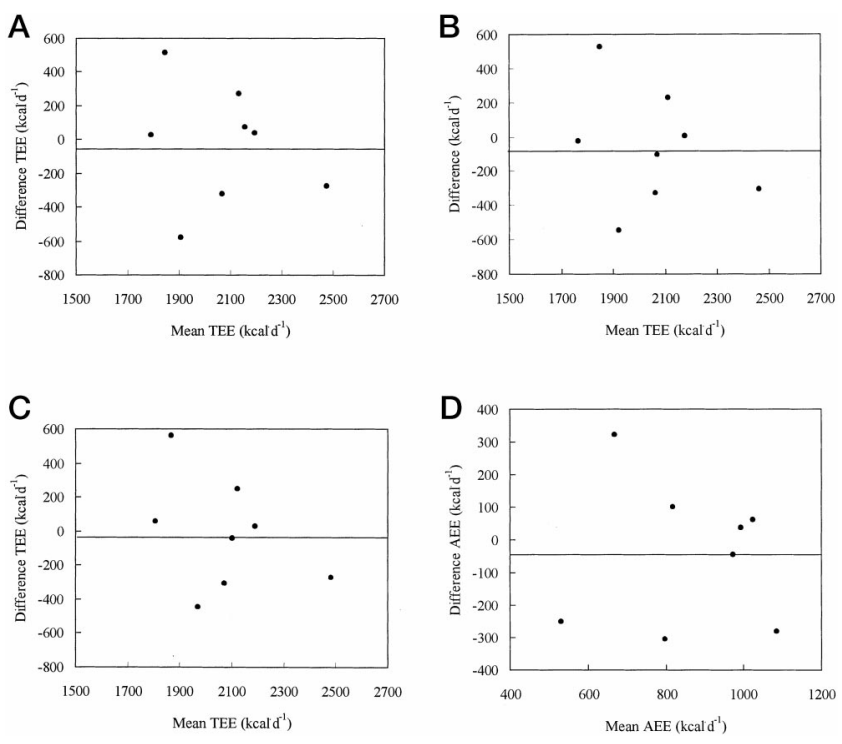

Figure 3-Cross validation of TEE and AEE prediction equations. The mean of predicted TEE for Regression 1 (gender, body weight, and activity counts) (A), Regression 2 (fat free mass, gender, and activity counts) (B), and Regression 3 (fat free mass, gender and activity counts) (C) and DLW-measured TEE is plotted against the difference between the methods. D. The mean of predicted AEE, Regression 4 (activity counts and gender), and DLW-measured AEE is plotted against the difference between the methods. tributed to the explained variation in all TEE regression models.

$\mathrm{AEE}$ is the most variable component of TEE (10). Although AEE and activity counts measure different dimensions of physical activity, i.e., AEE provides no information about the patterns of physical activity, they may be related. Given the cost and complexity of measuring AEE, alternative measures are warranted. Activity counts was significantly correlated to $\operatorname{AEE}(\mathrm{r}=0.54 ; P<0.01)$, and in a stepwise regression equation, was a unique predictor of AEE $\left(\mathrm{R}^{2}=0.16\right)$. Furthermore, $45 \%$ of the variation in AEE was explained by activity counts and gender. It has previously been suggested that $\dot{\mathrm{VO}}_{2 \max }$ could be used as a proxy indicator of AEE in children (11). Goran et al. (11) found that $35 \%$ of the variation in AEE could be explained by $\mathrm{VO}_{2 \max }$ and fat mass, derived from dual-energy x-ray absorbtiometry. Thus, the data from the present study indicate that an even better explained variation in AEE could be obtained using activity counts from the CSA activity monitor in combination with gender. The developed regression equations include anthropometric data together with activity monitor counts, simple measures that are feasible to obtain in large-scale epidemiological studies. It is suggested that the developed regression equations can be used for the prediction of TEE and AEE during free-living conditions in 9-yr-old children.

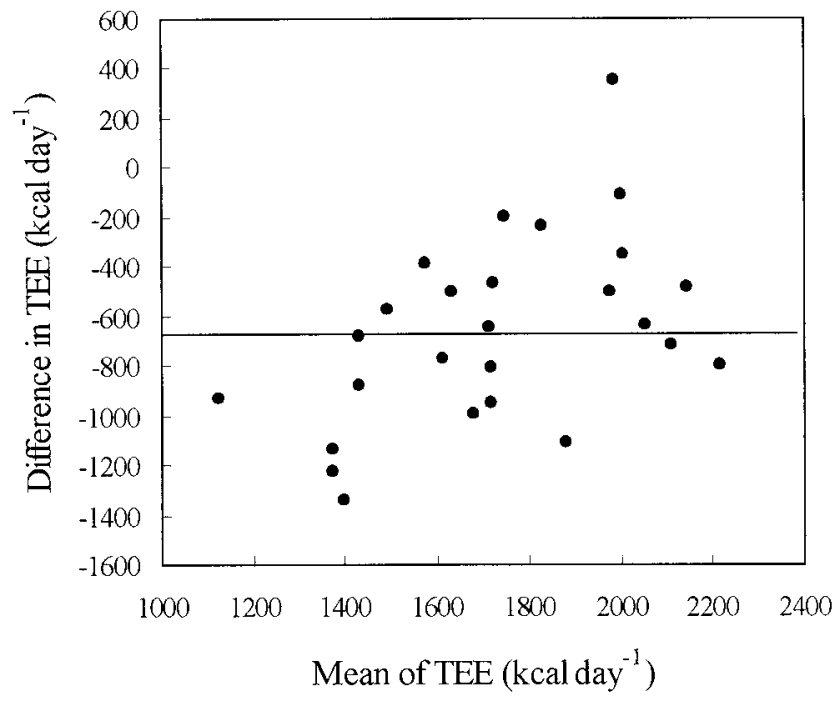

Figure 4-The association of TEE predicted from a laboratory-derived regression equation in combination with estimated BMR and TEE measured by DLW. The mean of the two methods are plotted against the difference between the methods (mean difference $=-658$ \left. $\pm 379{\mathrm{kcal} \cdot \mathrm{d}^{-1}}^{-1} P<0.001\right)$. 
The cross-validation study showed no significant differences between predicted and measured TEE and AEE for any of the developed prediction equations (Figure 3A-D). However, the relatively large SEE $\left(149 \mathrm{kcal} \cdot \mathrm{d}^{-1}\right.$ to 162 $\mathrm{kcal} \cdot \mathrm{d}^{-1}$ ) together with the wide limits of agreement preclude individual comparison. It is therefore suggested that the prediction equations could be used to assess the mean energy expenditure on a group level.

Although $45 \%$ of the variation in AEE and $60 \%$ of the variation in TEE was explained by activity counts, gender, and body weight, a discrepancy of $40-55 \%$ remains to be explained. At least part of this discrepancy is due to the function of the activity monitor. The CSA activity monitor is a uni-axial activity accelerometer measuring accelerations in the vertical plane. It is unlikely that a uni-axial accelerometer detects all human movement. Static work and movement against external forces, which result in an increase in energy expenditure without a proportional increase in the amount of body movement, is not detected by a uni-axial accelerometer. Furthermore, it has been shown that uni-axial accelerometers cannot account for increased energy expenditure due to inclines (16). Similarly, during bicycling, which is a common form of transportation in Danish children, the vertical movement of the body is not proportional to the increase in energy expenditure.

The use of prediction equations for calculation of BMR is a limitation of this study and may affect the results. However, these predictive equations are based on measurements in more than 700 children with a standard error of approximately $67 \mathrm{kcal}^{- \text {day }^{-1}}$ (24). A mean difference of $9.9 \%$ and $-2.7 \%$ between measured and predicted BMR has been reported in 9-yr-old children in UK and the Netherlands $(14,21)$. Despite the limitation of expressing AEE and PAL using calculated values for BMR, the results from the present study suggest that activity counts from the CSA activity monitor can be a useful indicator of the total amount of everyday physical activity in children, at least in the present age group.

To test the usefulness of a laboratory-derived predictive equation to assess TEE, data from the subjects in the present study were used. The published equation used to estimate average energy expenditure from the CSA activity monitor output was generated from Trost et al. (27). The equation underestimated TEE and demonstrated wide limits of agreement, indicating its unacceptability for predicting TEE in this group of children. The laboratoryderived prediction equation was established during walking and running on the treadmill. It is not likely that the relationship between activity counts and energy expenditure in a controlled laboratory setting reflects the relationship in free-living children. The nature of activities
TABLE 4. Correspondence between daily physical activity level (PAL $=$ TEE/BMR) and activity counts (counts·min ${ }^{-1}$ ) in discriminating between low, moderate, and high active groups.

\begin{tabular}{cccc}
\hline & \multicolumn{3}{c}{ Activity Counts } \\
\cline { 2 - 4 } & Low & Moderate & High \\
\hline PAL & 6 & & \\
Low & 3 & 4 & 1 \\
Moderate & 3 & 6 \\
High & & & 2 \\
\hline
\end{tabular}

performed by free-living children is much more complex and may explain the discrepancy. Furthermore, to calculate TEE, an estimated value for energy expenditure during sleep was added to EE from the predictive equation, which may contribute to the discrepancy. However, the large amount of time registered $\left(>13 \mathrm{~h} \cdot \mathrm{d}^{-1}\right)$ indicates that the activity monitor was worn by the children during most of the daytime. Therefore, it can be assumed that the vast majority of nonregistered time was sleeping time. This justifies the use of predicted BMR during nonregistered time. Nevertheless, the Trost et al. (27) equation may still be useful for estimating energy expenditure during walking and running in children.

When discriminating among separate levels of daily physical activity, CSA activity counts was not significantly different from PAL values. Although 10 of the 26 subject's activity level as indicated by the CSA activity monitor differed from that indicated by PAL, only one subject was classified in the high activity group by activity counts and in the low group by PAL (Table 4). The intervals used for PAL covers those recommended by Torun et al. (26) to indicate physical activity levels with low, moderate, and high levels of physical activity in this age group. The present results suggest that activity counts can be used to discriminate between high active and low active children.

In summary, activity counts from the CSA activity monitor seem to provide valid data for assessing the total amount of physical activity in 9-yr-old children. Regression equations for the prediction of total daily energy expenditure and activity energy expenditure from simple anthoprometric measures and activity counts were developed and cross validated in a random subsample of the children. Additional research is needed to develop comparable equations in other age groups of children and adolescents.

The authors express their appreciation to Malin Blanch and Caroline Griffiths for their technical assistance in Odense, to Loek Wouters for performing the isotope analysis in Maastricht, and to Olle Carlsson at Örebro University for invaluable statistical assistance. The public health committee of Stockholm's County Council funded this project.

Address for correspondence: Ulf Ekelund, Department of Physical Education and Health, University of Örebro, S-70182 Örebro, Sweden. E-mail: ulf.ekelund@ioh.oru.se. 


\section{REFERENCES}

1. Black, A. E., A. M. Prentice, and W. A. Coward. Use of food quotients to predict respiratory quotients for the doubly-labeled water method of measuring energy expenditure. Hum. Nutr. Clin. Nutr. 40:381-391, 1986.

2. Bland, J. M., and D. G. Altman. Statistical methods for assessing agreement between two methods of clinical measurement. Lancet 1:307-310, 1986.

3. Boreham, C. A., J. Twisk, M. J. Savage, G. W. Cran, and J. J. StRain. Physical activity, sports participation, and risk factors in adolescents. Med. Sci. Sports Exerc. 29:788-793, 1997.

4. Bouten, C. V. C., W. P. H. G. Verboeket-Van de Venne, K. R. Westerterp, M. Verduin, and J. D. Janssen. Daily physical activity assessment: comparison between movement registration and doubly labeled water. J. Appl. Physiol. 81:1019-1026, 1996.

5. Bratteby, L. E., B. Sandhagen, H. Fan, and G. Samuelson. A seven day activity diary for assessment of daily energy expenditure validated by the doubly labelled water method. Eur. J. Clin. Nutr. 51:585-591, 1997.

6. Computer Science and Applications Inc. Activity Monitor Operator's Manual, Model 7164, Release 1.21, Shalimar, FL: Computer Science and Applications Inc, 1997, pp. 4-5.

7. Eston, R. G., A. V. Rowlands, and D. K. Ingledew. Validity of heart rate, pedometry, and accelerometry for predicting the energy cost of children's activities. J. Appl. Physiol. 84:362-371, 1998.

8. Fomon, S. J., F. HaschKe, E. E. Ziegler, and S. E. Nelson. Body composition of reference children from birth to age 10 years. Am. J. Clin. Nutr. 35:1169-1175, 1982.

9. Freedson, P. S., E. Melanson, and J. Sirard. Calibration of the Computer Science and Applications, Inc. Accelerometer. Med. Sci. Sports Exerc. 30:777-781, 1998.

10. Goran M. I. Variation in total energy expenditure in humans. Obes. Res. 3:59-66, 1993

11. Goran, M. I., T. R. NAGy, B. A. Gower, et al. Influence of sex, seasonality, ethnicity, and geographic location on the components of total energy expenditure in young children: implications for energy requirements. Am. J. Clin. Nutr. 68:675-682, 1998.

12. Janz, K. F, J. WitT, and L. T. Mahoney. The stability of children's physical activity as measured by accelerometry and self-report. Med. Sci. Sports Exerc. 27:1326-1332, 1995.

13. Johnson, R. K., J. Russ, and M. I. Goran. Physical activity related energy expenditure in children by doubly labelled water as compared with the Caltrac accelerometer, Int. J. Obes. 22: 1046-1052, 1998.

14. Livingstone, M. B. E., W. A. Coward, A. M. Prentice, et al. Daily energy expenditure in free living children: comparison of heart rate monitoring with the doubly labelled water $\left({ }^{2} \mathrm{H}_{2}{ }^{18} \mathrm{O}\right)$ method. Am. J. Clin. Nutr. 56:343-352, 1992.

15. Maffeis, C., Y. Schutz, L. Zoccante, L. Micciolo, and L. Pinelli. Meal-induced thermogenesis in lean and obese prepubertal children. Am. J. Clin. Nutr. 57:481-485, 1993.
16. Melanson, E., and P. S. Freedson. Validity of the Computer Science and Applications, Inc. (CSA) activity monitor. Med. Sci. Sports Exerc. 27:934-940, 1995.

17. Murgatroyd, P., P. Shetty, and A. M. Prentice. Techniques for the measurement of human energy expenditure: a practical guide. Int. J. Obes. 17:549-568, 1993.

18. Raitakari, O. T., S. Taimela, K. V. PorkKa, et al. Associations between physical activity and risk factors for coronary heart disease (CHD): the Cardiovascular Risk in Young Finns Study. Med. Sci. Sports Exerc. 29:1055-1061, 1997.

19. Sallis, J. F., M. J. Buono, J. J. Roby, D. Carson, and J. A. Nelson. The Caltrac accelerometer as a physical activity monitor for school-aged children. Med. Sci. Sports Exerc. 22:698-703, 1990.

20. Saluis, J. F., and N. Owen. In: Physical Activity and Behavioral Medicine. Thousand Oaks, CA: Sage Publications, 1999, pp. 79-83.

21. Saris, W. H. M., H. J. G. Emons, D. C. Groenenboom, and K. R. WesterterP. Discrepancy between FAO/WHO energy requirements and actual energy expenditure in healthy 7-11 year old children (Abstract). In: Children and Exercise. Stuttgart: Enke, 1990, p. 119.

22. Schoeller, D. A. Measurement of energy expenditure in free living humans by using doubly labeled water. J. Nutr. 118:12781289, 1988.

23. Schoeller, D. A, and S. B. Racette. A review of field techniques for the assessment of energy expenditure. J. Nutr. 120:1492-1495, 1990.

24. Schofield, W. N. Predicting basal metabolic rate, new standards and review of previous work. Hum. Nutr. Clin. Nutr. 39(Suppl. 1):5-41, 1985.

25. Slaughter, M. H., T. G. Lohman, R. A. Boileau, et al. Skinfold equations for estimation of body fatness in children and youth. Hum. Biol. 60:709-723, 1988.

26. Torun, B., P. S. W. Davies, M. B. E. Livingstone, M. Paolisso, R. SACKETT, and G. B. SpurR. Energy requirements and dietary energy recommendations for children and adolescents 1 to 18 years old. Eur. J. Clin. Nutr. 50(Suppl. 1):37-81, 1996.

27. Trost, S. G, D. S. Ward, S. M. Moorehead, P. D. Watson, W. RINER, and J. R. BURKE. Validity of the computer and science applications (CSA) activity monitor in children. Med. Sci. Sports Exerc. 30:629-633, 1998.

28. TRYon, W. W., and R. Williams. Fully proportional actigraphy: a new instrument. Behav. Res. Methods Instrum. Comp. 28:392403, 1996.

29. Westerterp, K. R., F. Brouns, W. H. M. Saris, and F. Ten Hoor. Comparison of doubly labeled water with respspirometry at lowand high-activity levels. J. Appl. Physiol. 65:53-56, 1988.

30. Westerterp, K. R., L. Wouters, and W. D. Van Marken LichtENBELT. The Maastricht protocol for the measurement of body composition and energy expenditure with labeled water. Obes. Res. 3(Suppl.1):49-57, 1995. 